\title{
Germline HOXB13 p.Gly84Glu mutation and cancer susceptibility: a pooled analysis of 25 epidemiological studies with 145,257 participates
}

\author{
Qiliang Cai ${ }^{1}$, Xinpeng Wang ${ }^{1}$, Xiaodong Li $^{2}$, Rui Gong ${ }^{3}$, Xuemei Guo ${ }^{4}$, Yang Tang ${ }^{1}$, \\ Kuo Yang ${ }^{1}$, Yuanjie Niu' ${ }^{1}$, Yan Zhao ${ }^{5}$ \\ ${ }^{1}$ Department of Urology, Tianjin Institute of Urology, the Second Hospital of Tianjin Medical University, Tianjin, 300211, \\ China \\ ${ }^{2}$ Department of Radiotherapy, the Second Hospital of Tianjin Medical University, Tianjin, 300211, China \\ ${ }^{3}$ Pharmaceutical Department, the Second Hospital of Tianjin Medical University, Tianjin, 300211, China \\ ${ }^{4}$ Library of Tianjin Medical University, Tianjin Medical University, Tianjin, 300070, China \\ ${ }^{5}$ Tianjin Institute of infectious diseases, the Second Hospital of Tianjin Medical University, Tianjin, 300211, China \\ Correspondence to: \\ Yan Zhao, e-mail: zhaoyan2010109@163.com \\ Keywords: HOXB13 gene, rs 138213197, genetic mutation, cancer, risk \\ Received: August 01, $2015 \quad$ Accepted: October 05, $2015 \quad$ Published: October 17, 2015
}

\section{ABSTRACT}

Numerous studies have investigated association between the germline HOXB13 p.Gly84Glu mutation and cancer risk. However, the results were inconsistent. Herein, we performed this meta-analysis to get a precise conclusion of the associations. A comprehensive literature search was conducted through Medline (mainly Pubmed), Embase, Cochrane Library databases. Crude odds ratios (ORs) and their 95\% confidence intervals (CIs) were calculated by STATA 12.1 software to evaluate the association of HOXB13 p.Gly84Glu mutation and cancer susceptibility. Then, 25 studies including 51,390 cases and 93,867 controls were included, and there was significant association between HOXB13 p.Gly84Glu mutation and overall cancer risk $(O R=2.872,95 \% C I=2.121-3.888, P<0.001)$, particularly in prostate cancer $(\mathrm{OR}=3.248,95 \% \mathrm{CI}=2.313-4.560, P<0.001)$, while no association was found in breast $(O R=1.424,95 \% C I=0.776-2.613, P=0.253)$ and colorectal cancers $(O R=$ $2.070,95 \% \mathrm{CI}=0.485-8.841, P=0.326)$. When we stratified analysis by ethnicity, significant association was found in Caucasians $(O R=2.673,95 \% C I=1.920-3.720$, $P<0.001)$. Further well-designed with large samples and other various cancers should be performed to validate our results.

\section{INTRODUCTION}

Cancer is a serious problem endangering the human health and lives. Based on the reports from International Agency for Research on Cancer (IARC), cancer has become the second leading cause of mortality in developing countries, which has exceeded the mortality caused by cardiovascular incidences and become the leading cause of mortality in developed countries $[1,2]$. Totally, 1,658,370 new cancer cases were diagnosed and 589,430 patients died from cancer in the United States in 2015 [3], suggesting that the burden of cancer will be heavier year by year, due to the increasing number of world population and the problem of aging is getting worse [4]. Although the mechanism of carcinogenesis remains elusive, multiple environmental and lifestyle factors has been confirmed contributed to the formation of cancers. However, not all cancer patients who have been exposed to the risk factors will develop cancer, suggesting the inter-individual differences in susceptibility [5]. Therefore, genetic, environmental and life time factors were suggested to be the main determinant of individual risk for cancer [6, 7]. In recent years, numerous studies have pointed out genetic factors, particularly single nucleotide polymorphisms (SNPs) of genes, plays crucial roles in tumorigenesis [8-11]. 
HOXB13, which encodes the transcription factor 13, belongs to the $\mathrm{HOXB}$ gene cluster at chromosome 17 [12], involves in embryonic development of different organs [13], regulates transcription of androgen receptor (AR) target genes [14] and is reported to function as a tumor suppressor in cancer [15]. Deregulation of HOXB13 expression has been reported in a number of malignancies, including prostate, breast, colon, lung, endometrial, renal cancers and melanoma [16-19]. Recently, a novel germline mutation, p.Gly84Glu (rs138213197), in exon one of the HOXB13gene, was suggested to have a close relationship with the risk of various cancers. Numerous studies have focused on the association between the germline HOXB13 p.Gly84Glu mutation and cancer risks, however, the results are inconsistent. Thus, we comprehensively searched all related literatures and performed present meta-analysis which has great power through polling all eligible related data to get a more precise conclusion.

\section{RESULTS}

\section{Characteristics of included studies}

Figure 1 represents the process of eligible studies' identification and selection. The literature selective process was conducted rigorously according to the inclusion and exclusion criteria. Finally, 15 publications involving 25 individual studies with 51,390 cases and 93,867 controls were included in the present meta-analysis [20-34]. The main characteristics of included studies were summarized in Table 1. These studies included 19 prostate cancer studies, 3 breast cancer studies and 3 colorectal cancer studies. OF the 25 studies, there were 21 studies of Caucasian, and the other four studies of mixed ethnicity (both of them were the mixed population of Caucasian, Asian and African-American). As for the control source, five studies applied population-based (PB) control, 14 studies employed hospital-based (HB) control, four
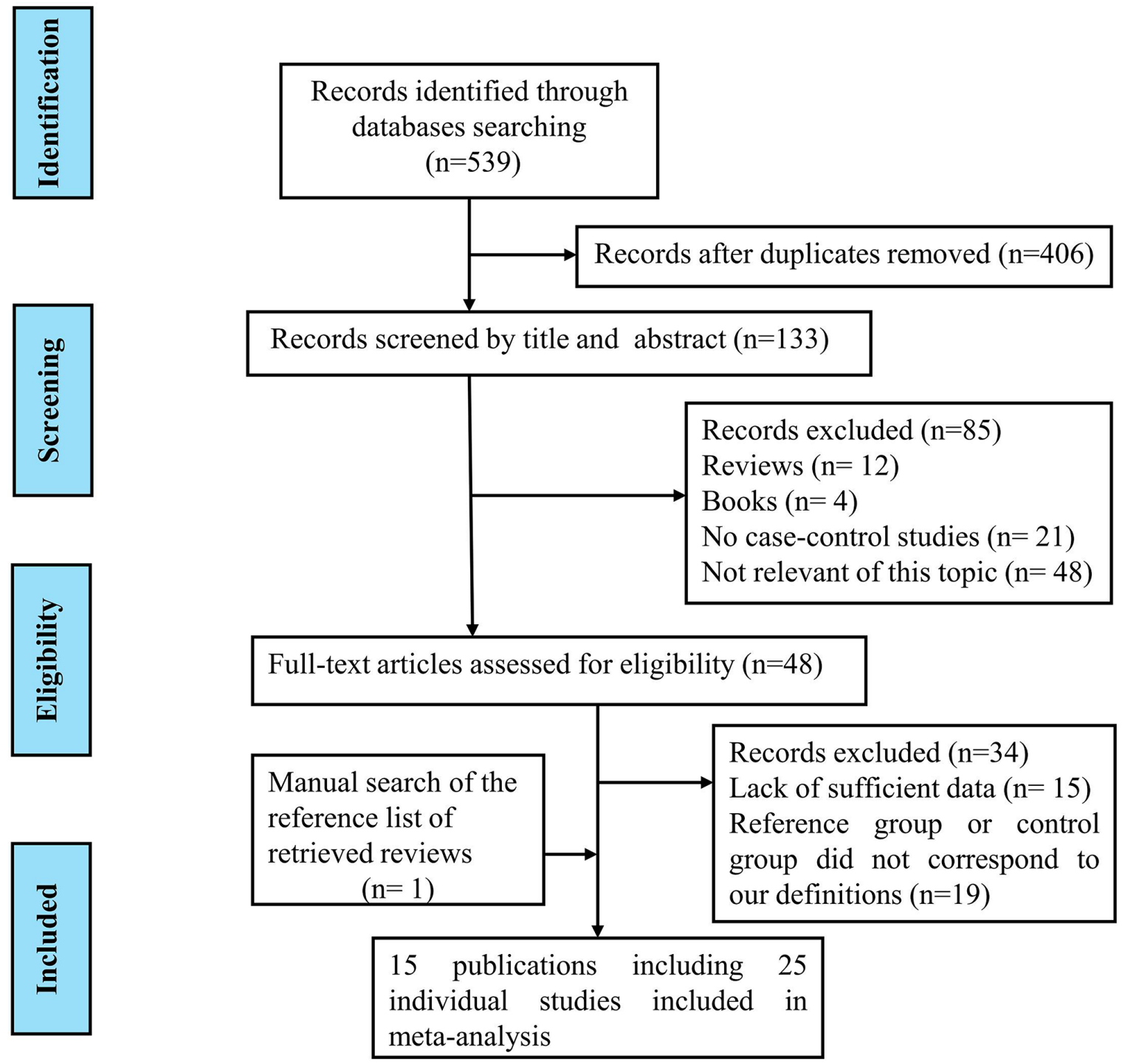

Figure 1: Flow diagram of summarizing the search strategy. 


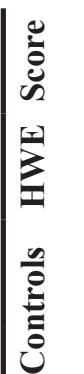
a

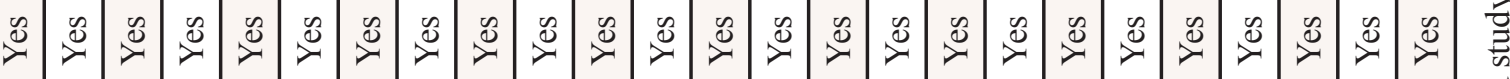

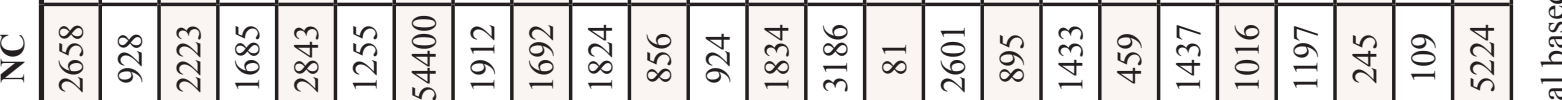

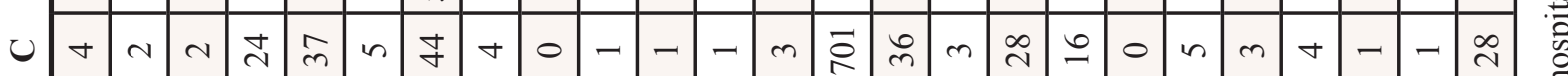

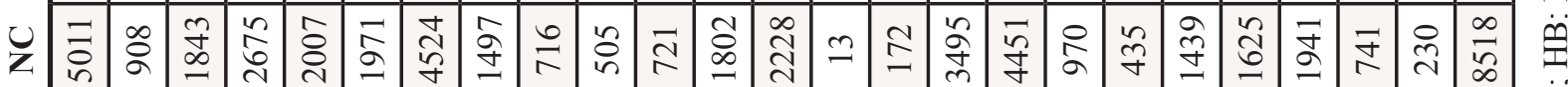

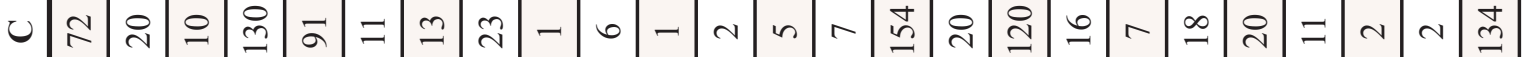

ช

药

竞

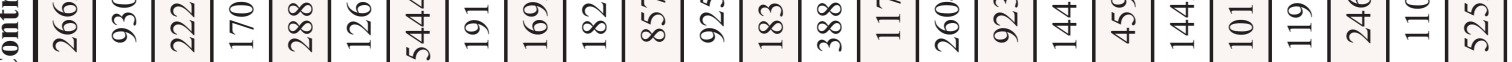

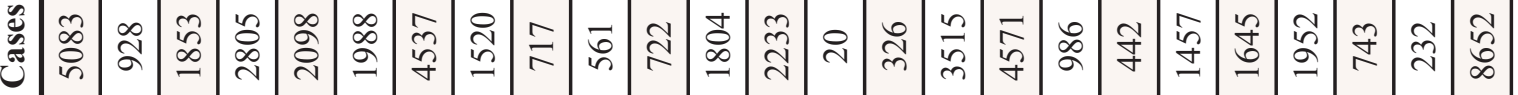

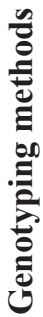

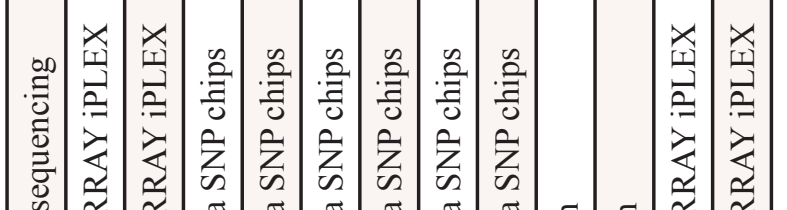

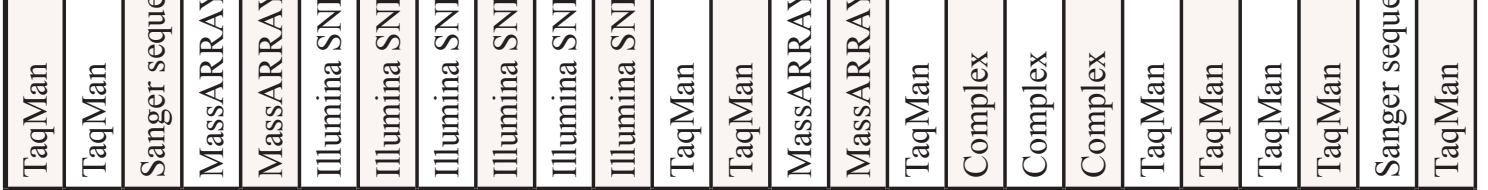

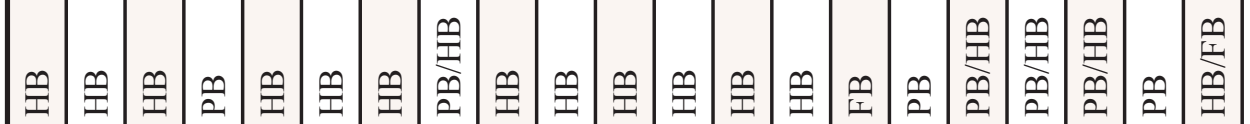

.

U

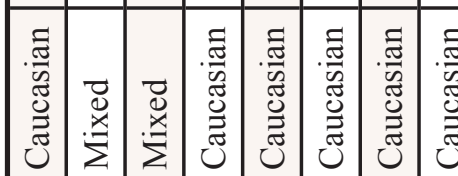

志

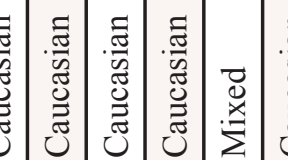

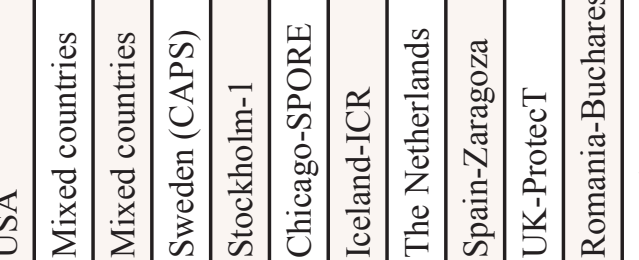

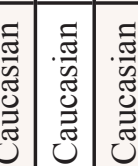

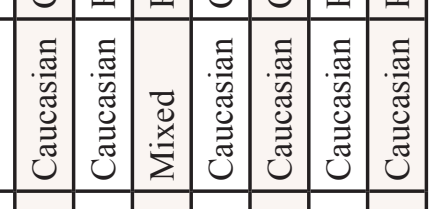

ป็

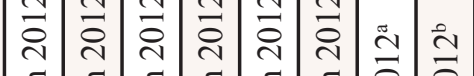

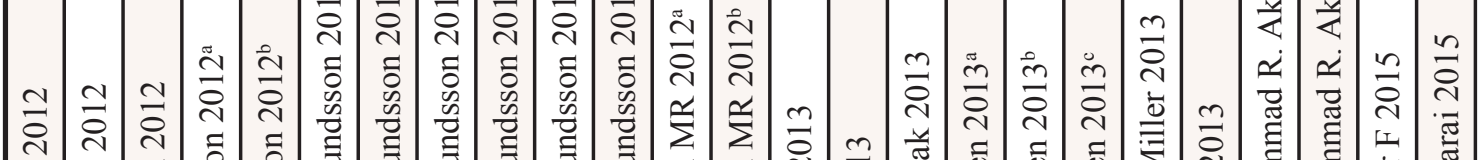

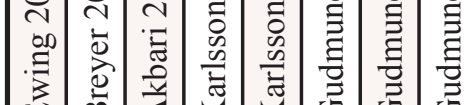

:

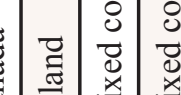

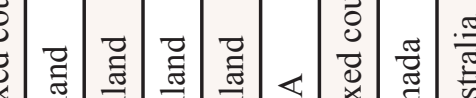

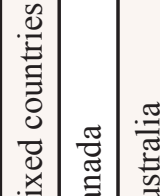

芯弚

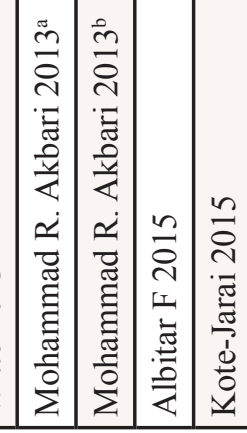

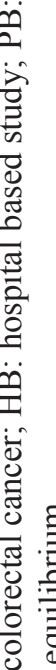

$\because$ on

$\cup$ :

8

ฮृ

范

பั

记

क⿺⿻一⿰冫⿰亅⿱丿丶丶⿱⿰㇒一乂

苍

鿒.

䒿

을 荅

क in

ब

:

乙.

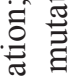

芫

苟

$\Xi \bar{\nabla}$

\%

总 글

는

远

$=$

䒕

过

这

i. 
studies applied PB/HB control, one studies applied familybased (FB) control, while the other one applied HB/FB control. Simultaneously, various genotyping methods were employed of all included studies, such as, 10 studies applied TaqMan assay, two studies applied Sanger sequencing, four studies applied MassARRAY iPLEX, six studies applied Illumina SNP, and the rest three studies employed complex methods (TaqMan, MassARRAY iPLEX, Sanger sequencing). The genotype distributions of all included studies in this meta-analysis were in agreement with Hardy-Weinberg equilibrium (HWE). The estimated quality of all included studies was in the range of 7-9 scores and was listed in Table 1.

\section{Quantitative data analyses}

Finally, 25 epidemiological individual studies including 51,390 cases and 93,867 controls were enrolled in this meta-analysis. There is significant heterogeneity was found in over cancer risk estimation $\left(I^{2}=62.8 \%, \quad \mathrm{P}_{\text {heterogeneity }}<0.0001\right) . \quad$ Considering that, random-effects model was used to examine the association between HOXB13 p.Gly84Glu mutation and overall cancer susceptibility $(\mathrm{OR}=$ 2.872, 95\% CI $=2.121-3.888, P<0.001$; Figure 2). In order to detect the source of heterogeneity, subgroup analyses were conducted by cancer type, ethnicity, control source and genotyping method. When we stratified by cancer type, there is significant heterogeneity was existed $\left(I^{2}=68.4 \%, \mathrm{P}_{\text {heterogeneity }}<0.0001\right)$ and then random-effect models was used to verify the relationship between HOXB13 p.Gly84Glu mutation and prostate cancer risk. The results presented that HOXB13 p.Gly84Glu mutation contributed to the susceptibility of prostate cancer $(\mathrm{OR}=3.248,95 \% \mathrm{CI}=2.313-4.560, P<0.001$; Figure 3). While no heterogeneities (for breast cancer: $I^{2}=0.0 \%, \mathrm{P}_{\text {heterogeneity }}=0.958$; and for colorectal cancer: $I^{2}=36.9 \%, \mathrm{P}_{\text {heterogeneity }}=0.205$, respectively) were found

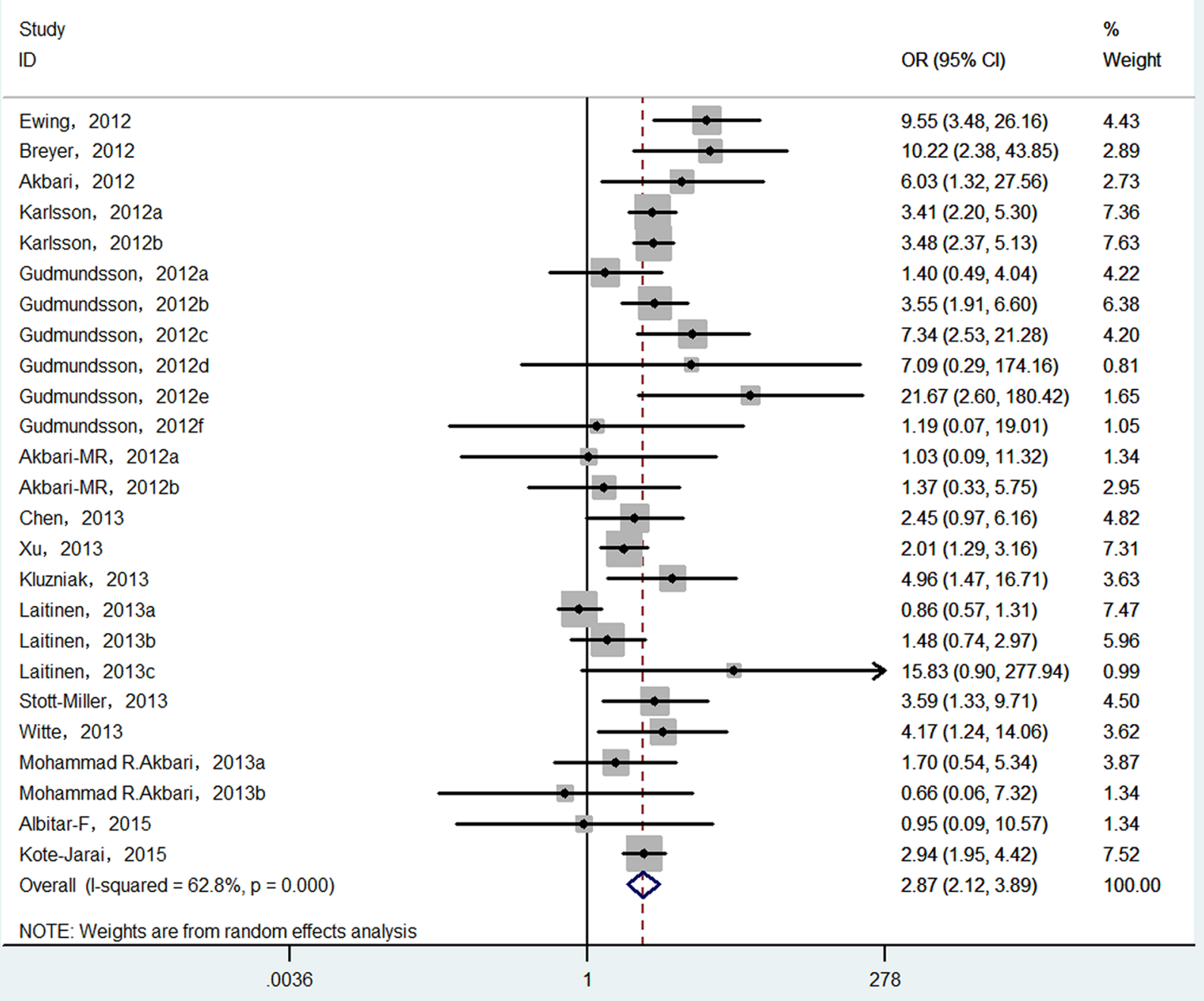

Figure 2: Forest plot of overall cancer risk associated with HOXB13 p.Gly84Glu mutation. 
Study

ID

OR $(95 \% \mathrm{Cl})$

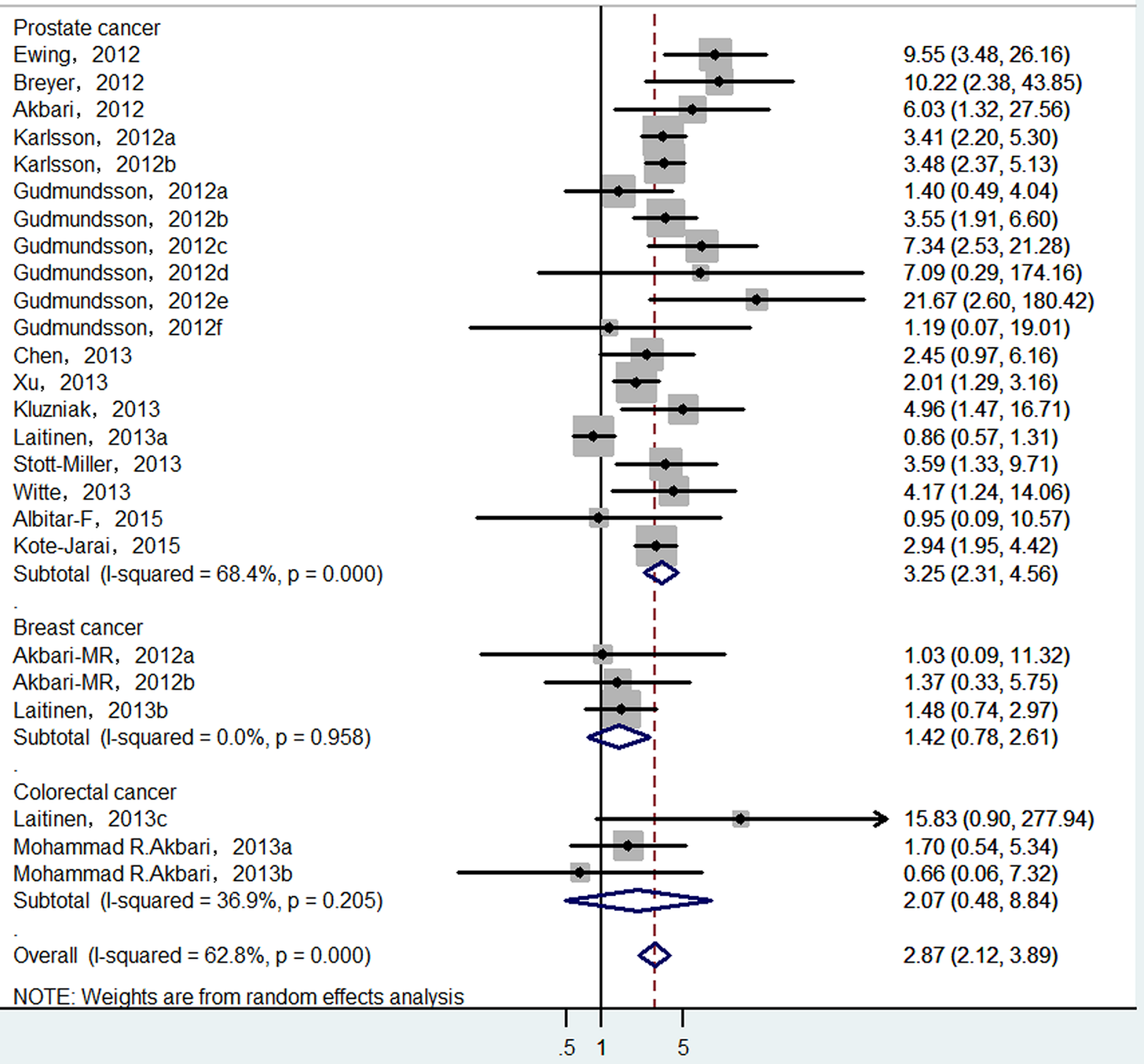

Figure 3: Forest plot of prostate cancer risk associated with HOXB13 p.Gly84Glu mutation.

when we analyzed the association between HOXB13 p.Gly84Glu mutation and the risk of the other two kinds of cancers mentioned above. HOXB13 p.Gly84Glu mutation was not contributed to the development of breast cancer $(\mathrm{OR}=1.423,95 \% \mathrm{CI}=0.774-2.615$, $P=0.256$; Table 2) and colorectal cancer $(\mathrm{OR}=2.458$, $95 \% \mathrm{CI}=0.98-6.177, P=0.056$; Table 2) using fixedeffect models. Moreover, further subgroup analyses were also performed by study design and genotyping method. All the results of meta-analyses were summarized in Table 2.

\section{Sensitivity analyses}

One-way sensitivity analysis of the pooled OR and $95 \%$ CIs for HOXB13 p.Gly84Glu was performed to verify if the results of our present meta-analysis were robust. The pooled ORs were calculated by means of a random effects model. To the best of our knowledge, in a sensitivity analysis, if a single study included in a metaanalysis was omitted each time, the pooled ORs were always persistent and it can be considered as the results of this meta-analysis were reliable and stable. In the present 
Table 2: Meta-analyses results of the association between germline HOXB13 p.Gly84Glu mutation and cancer risk

Variables
\begin{tabular}{|l|c|c|c|c|c|c|c|}
\hline Total & 25 & 145,257 & $<0.001$ & Random & 2.872 & $2.121,3.888$ & $<0.001$ \\
\hline Cancer type & & & & & & & \\
\hline Prostate cancer & 19 & 130,795 & $<0.001$ & Random & 3.248 & $2.313,4.560$ & $<0.001$ \\
\hline Breast cancer & 3 & 9,423 & 0.958 & Fixed & 1.423 & $0.774,2.615$ & 0.256 \\
\hline Colotrectal cancer & 3 & 5,039 & 0.205 & Fixed & 2.458 & $0.978,6.177$ & 0.056 \\
\hline Ethnicity & & & & & & & \\
\hline Caucasians & 21 & 144,007 & $<0.001$ & Random & 2.673 & $1.920,3.720$ & $<0.001$ \\
\hline Mixed decedents & 4 & 12,507 & 0.362 & Fixed & 4.164 & $2.226,7.790$ & $<0.001$ \\
\hline Genotype method & & & & & & & \\
\hline TaqMan & 10 & 46,126 & 0.149 & Fixed & 3.649 & $2.728,4.880$ & $<0.001$ \\
\hline Sanger sequencing & 2 & 4,420 & 0.201 & Fixed & 3.862 & $1.110,13.441$ & 0.034 \\
\hline MassARRAY iPLEX & 4 & 13,842 & 0.201 & Fixed & 2.956 & $2.337,3.740$ & $<0.001$ \\
\hline Illumina SNP chips & 6 & 72,039 & 0.135 & Fixed & 3.934 & $2.479,6.245$ & $<0.001$ \\
\hline Complex methods & 3 & 8,830 & 0.067 & Fixed & 1.119 & $0.784,1.597$ & 0.537 \\
\hline Source of control & & & & & & & \\
\hline Hospital based -HB & 14 & 112,986 & 0.155 & Fixed & 3.363 & $2.449,4.619$ & $<0.001$ \\
\hline Population based -PB & 5 & 17,670 & 0.481 & Fixed & 3.196 & $2.234,4.573$ & $<0.001$ \\
\hline PB/HB & 4 & 11,494 & 0.001 & Random & 2.378 & $0.814,6.952$ & 0.113 \\
\hline Family based -FB & 1 & 443 & - & Fixed & 2.015 & $1.286,3.156$ & 0.002 \\
\hline HB/FB & 1 & 2,664 & - & Fixed & 4.168 & $1.235,14.062$ & 0.021 \\
\hline
\end{tabular}

OR, Odds ratio; 95\% CI, 95\% confidence interval.

meta-analysis, no single study was qualitatively influenced by the pooled ORs when they were sequentially omitted, as indicated by the sensitivity analyses, suggesting that the results of our present study are stable (Figure 4).

\section{Publication bias}

Begg's tests were employed to detect the potential publication bias that may be existed in this meta-analysis, and the results suggested there was no publication bias $(P=0.815$, Figure 5a). Egger's tests also confirmed the absence of publication bias in the meta-analysis $(P=0.30$, Figure 5b).

\section{DISCUSSION}

Here, we conducted the largest meta-analysis to summarize the association between HOXB13 p.Gly84Glu mutation and cancer risk through pooling all candidate studies. Totally, 25 epidemiological case-control studies including 51,390 cases and 93,867 controls were enrolled this present study. In recent years, HOXB13 p.Gly84Glu mutation was found to contributed to the risk of prostate cancer [20-24, 26-31, 33, 34], especially in European countries [21]. However, no significant association was found in Asians [22] and Africans [21, 22]. Additionally, there are also three studies from two publications reported the association and they got the final conclusion that this mutation was not found to have increased the susceptibility of breast cancer on both familial and sporadic patients $[25,29]$. Similarly, no significant association was detected among the mutation and the risk of colorectal cancer $[29,32]$. In our present study, we summarized all the effects of HOXB13 p.Gly84Glu mutation with the risk of various cancers. We got a conclusion that the mutation is significantly increased the risk of cancers. Then, we performed subgroup analyses according to cancer type, ethnicity, text method, et al. We found that the mutation is contributed to the risk of prostate cancer, which was in accordance with previous studies. Moreover, we failed 


\section{Meta-analysis random-effects estimates (exponential form)}

\section{Study ommited}

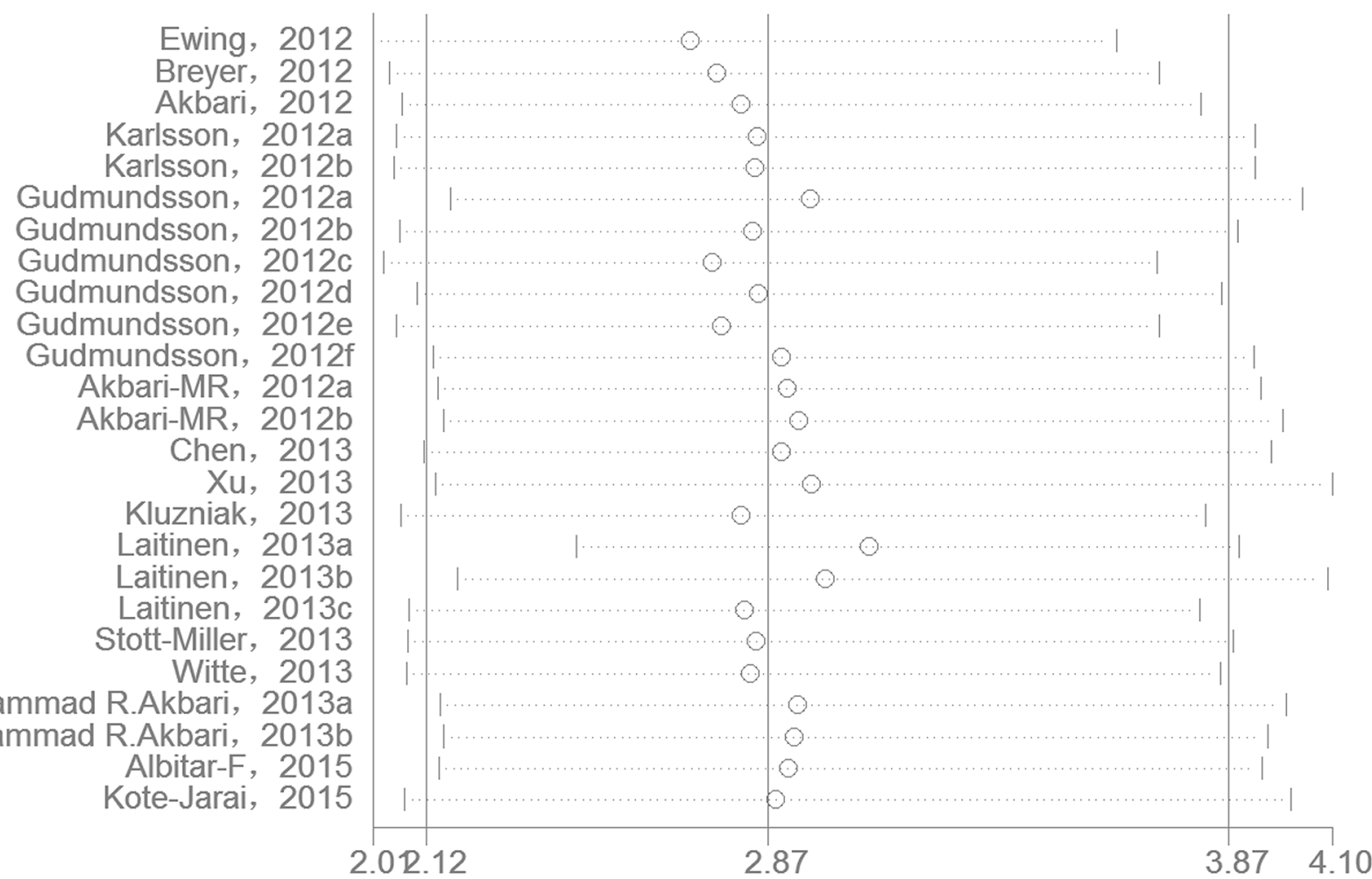

Figure 4: One-way sensitivity analysis of the pooled ORs and 95\% CI for HOXB13 p.Gly84Glu mutation, omitting each data set in the meta-analysis.

a: Publication bias was examined by Begg's test.

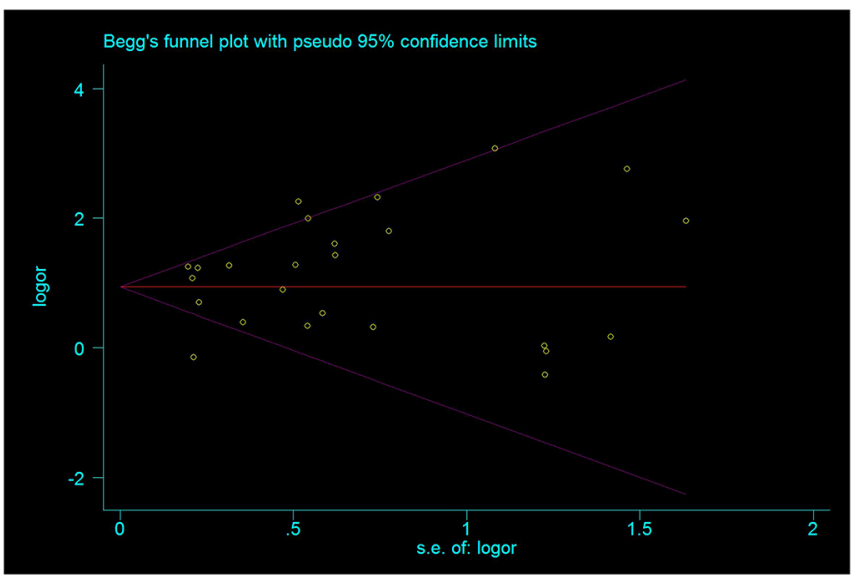

b: Publication bias was examined by Egger's test.

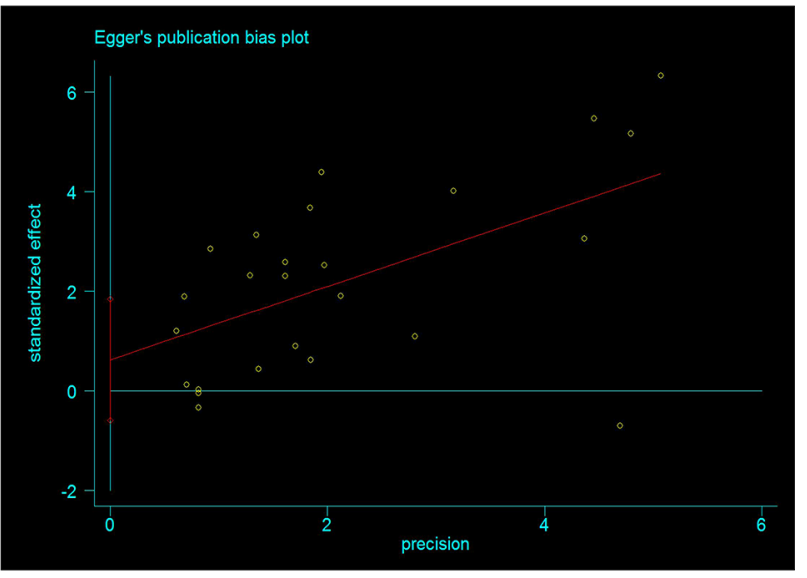

Figure 5: Publication bias was detected by Begg's (a) and Egger's (b) test.

to find a statistical association between the mutation and the susceptibility of breast cancer and colorectal cancer. As such, the results were similar with the previous studies mentioned above. Moreover, stratified analyses by ethnicity were performed, and the conclusion suggested that the mutation can increase the risk of overall cancer among Caucasians, particularly in European decedent patients.

Substantial heterogeneity between studies was existed in our meta-analysis, just as a common aspect of genetic association studies. We determined the heterogeneity by Q-test and $I^{2}$ test, and statistically 
significant heterogeneity was observed. Then, randomeffects model was used to analyze the ORs with 95\% CI. Although we performed the present study according to the PRISAM strictly, including strict criteria of selection publications and meta-regression performance, there was no source of heterogeneity found in our present meta-analysis. Therefore, we carried out subgroup meta-analyses, and the results suggested that these parameters including ethnicity, cancer type, control source and genotyping method may be the main source of heterogeneities. In the stratified analysis by genotype method, the heterogeneity was significantly reduced, suggesting that genotype method may be one of source of heterogeneities.

To our knowledge, meta-analysis has great power through pooling all eligible studies, and thus gets a reliable and relative precise result. In the present study, there are several advantages existed. Above all, this is by far an analysis with the largest sample size, which can make our result more reliable and precise. What's more, the quality of each study include study was high, ranged from 7-9 score. Moreover, one-way sensitivity analysis was performed, and the result suggested that no significant influence of a single study on the pooled ORs and $95 \%$ CI. Simultaneously, no significant publication bias was detected in our present work. Of the two factors mentioned above, it demonstrated that our results were stability and reliable. In addition, subgroup analyses were conducted to explore the association of HOXB13 p.Gly84Glu mutation and susceptibility to the three types of cancers.

Some limitations existed and should be acknowledged in the meta-analysis. On the one hand, there were only three types of cancers including prostate cancer, breast cancer and colorectal cancers. Based on that, the results of this present work may not have enough power to represent all kinds of cancer. On the other hand, most of studies included in this meta-analysis were of European and USA decedents belonged to Caucasian ethnicity, which was a cause of selection bias, and other ethnicities, such as, African, and Asian ethnicities should be included in further studies. In addition, even though no sample size and language limitations were set, related studies in other languages may be ignored. What's more, only published studies were included in this meta-analysis, while other unpublished studies in different languages should be enrolled. Finally, adjusted estimations were not performed for insufficient data, such as, age, sex, smoking and drinking habits et al which can interrupt the results of present study. Simultaneously, interactions among genegene, gene-environment, and even different polymorphism loci of the same gene were not conducted for lacking of sufficient data in this work, which may regulate the gene expression, affect the function of gene product, and lead to the different OR values. Thus, further studies with same topics should consider the factors mentioned above.
In summary, the meta-analysis suggested that HOXB13 p.Gly84Glu mutation contributed to the overall cancer risk, especially for prostate cancer. Considering limitations mentioned above, further well-designed studies with larger sample size should be conducted to verify the results of the present meta-analysis.

\section{MATERIALS AND METHODS}

The present meta-analysis was performed according to the latest meta-analysis guidelines (PRISMA) [35].

\section{Search strategy}

A comprehensive computerized literature search was conducted through Medline (main Pubmed), Embase, Cochrane Library, Web of Science, Wanfang and China National Knowledge Infrastructure (CNKI) databases for related research studies reported the association between HOXB13 p.Gly84Glu mutation and cancer susceptibility. Furthermore, we also searched related studies manually from the references of reviews and articles reported the same topics. Combinations of searching terms were used as follows: "HOXB13 p.Gly84Glu", "HOXB13 rs138213197”, “single nucleotide polymorphism, SNP or variation, mutation" and "cancer or carcinoma or tumor or neoplasms". In order to get a precise conclusion, no sample size and language limitations were set, hoping that we can identify all the studies that examined the association of HOXB13 p.Gly84Glu mutation and cancer risk.

\section{Inclusion and exclusion criteria}

The following criteria should be met of each included studies. (1) reported the association between HOXB13 p.Gly84Glu mutation and cancer risk; (2) used case-control design; (3) all the patients in cases group should be diagnosed by histochemical results or other gold diagnostic standers; (4) provided sufficient data of HOXB13 p.Gly84Glu mutation carriers or non-carriers, or other information such as ORs with 95\% CIs for statistical analysis; (5) if there were several publications with overlapping data, only the latest one with the largest sample size was finally included in this present work. At the same time, if each of the searched studies was in accordance with the following criteria, it must be excluded. (1) human being studies; (2) review, meeting or other types of abstracts, comment, correspondence, letters or letters to the editor; case reports, or case-only studies; (3) not provided the sufficient data to extract.

\section{Data extraction}

Two independent investigators extracted the essential information according to the selection criteria mentioned above. The key data was listed as follows: 
the first author's surname, year of publication, country of origin, ethnicity, cancer type, control source [population based (PB) or hospital based (HB)], the total number of cases and controls, genotyping methods, the number of HOXB13 p.Gly84Glu mutation carriers and non-carriers. Any disagreement was resolved by discussion, if not, other authors will join the discussion until consensus was reached.

\section{Statistical analysis}

Crude odds ratio (OR) with corresponding 95\% confidence interval $(95 \% \mathrm{CI})$ were used to calculated to assess the strength of the association between the HOXB13 p.Gly84Glu mutation and cancer risk. Heterogeneity was evaluated by $I^{2}$ test, with the $I^{2}$ value ranged from 0 to $100 \%: I^{2}=0-25 \%$ : no heterogeneity; $I^{2}=25-50 \%$ : moderate heterogeneity; $I^{2}=50-75 \%$ : large heterogeneity; $I^{2}=75-100 \%$ : extreme heterogeneity) $[36,37]$, and Cochrane $\mathrm{Q}$ test $(P<0.10$ represents a significant heterogeneity was existed). Both fixed-effects (Mantel-Haenszel) and random-effect (Der Simonian and Laird) models were used to analyze the pooled ORs. The fixed-effects model was used when there was no heterogeneity; otherwise, the random-effects model will be used [38, 39]. Subgroup analyses were conducted based on cancer type, control source, and geographical region, to determine the source of heterogeneity. One-way sensitivity analyses were also performed to evaluate the influence of each included study to the results of present meta-analysis. An estimation of potential publication bias was carried out by the Begg's and Egger tests. All the key parameters were calculated using STATA software (version 12.0; Stata Corporation, College Station, TX, USA). All the tests were two-sided, a $P$ value of less than 0.05 for any test or model was considered to be statistically significant.

\section{CONFLICTS OF INTEREST}

The authors have no conflicts of interest to declare.

\section{FUNDING}

This work was supported in part by Science and technology fund of Tianjin city health and Family Planning Commission (Grant No: 2014KZ093).

\section{REFERENCES}

1. Siegel R, Ma J, Zou Z, Jemal A. Cancer statistics, 2014. CA Cancer J Clin. 2014; 64:9-29.

2. Ferlay J, Shin HR, Bray F, Forman D, Mathers C, Parkin DM. Estimates of worldwide burden of cancer in 2008: GLOBOCAN 2008. Int J Cancer. 2010; 127:2893-2917.
3. Rebecca L. Siegel, Kimberly D. Miller, Ahmedin Jemal, DVM. Cancer Statistics, 2015. CA Cancer J Clin. 2015; 65:5-29.

4. Massagué J. TGF $\beta$ in cancer. Cell. 2008; 134:215-230.

5. Qiliang Cai, Zhun Wang, Wei Zhang, Xuemei Guo, Zhiqun Shang, Ning Jiang, Jing Tian, Yuanjie Niu. Association between glutathione S-transferases M1 and T1 gene polymorphisms and prostate cancer risk: a systematic review and meta-analysis. Tumor Biol. 2014; 35:247-256.

6. Wilson S, Jones L, Coussens C, Hanna K. (Eds.). Cancer and the environment: Gene-environment interaction, (National Academy Press, Washington, DC, 2002.

7. Qiliang Cai, Tao Wu, Wei Zhang, Xuemei Guo, Zhiqun Shang, Ning Jiang, Jing Tian, Yuanjie Niu. Genetic polymorphisms in glutathione S-transferases P1 (GSTP1) Ile105Val and prostate cancer risk: a systematic review and meta-analysis. Tumor Biol. 2013; 34:3913-3922.

8. Marshall AL, Christiani DC. Genetic susceptibility to lung cancer-light at the end of the tunnel. Carcinogenesis. 2013; 34:487-502.

9. Bartsch H, Dally H, Popanda O, Risch A, Schmezer P. Genetic risk profiles for cancer susceptibility and therapy response. Recent Results Cancer Res. 2007; 174:19-36.

10. Bozina N, Bradamante V, Lovrić M. Genetic polymorphism of metabolic enzymes P450 (CYP) as a susceptibility factor for drug response, toxicity, and cancer risk. Arh Hig Rada Toksikol. 2009; 60:217-42.

11. He XF, Wei W, Liu ZZ, Shen XL, Yang XB, Wang SL, Xie DL. Association between the CYP1A1 T3801C polymorphism and risk of cancer: evidence from 268 casecontrol studies. Gene. 2014; 534:324-344.

12. Zeltser L, Desplan C, Heintz N. Hoxb-13: a new Hox gene in a distant region of the HOXB cluster maintains colinearity. Development. 1996; 122:2475-84.

13. Kawazoe Y, Sekimoto T, Araki M, Takagi K, Araki K, Yamamura K. Region-specific gastrointestinal Hox code during murine embryonal gut development. Dev Growth Differ. 2002; 44:77-84.

14. Norris JD, Chang CY, Wittmann BM, Kunder RS, Cui H, Fan D, Joseph JD, McDonnell DP. The homeodomain protein HOXB13 regulates the cellular response toandrogens. Mol Cell. 2009; 36:405-416.

15. Hamid SM, Cicek S, Karamil S, Ozturk MB, Debelec-Butuner B, Erbaykent-Tepedelen B, Varisli L, Gonen-Korkmaz C, Yorukoglu K, Korkmaz KS. HOXB13 contributes to $\mathrm{G} 1 / \mathrm{S}$ and $\mathrm{G} 2 / \mathrm{M}$ checkpoint controls in prostate. Mol Cell Endocrinol. 2014; 383:38-47.

16. Cantile M, Pettinato G, Procino A, Feliciello I, Cindolo L, Cillo C. In vivo expression of the whole HOX gene network in human breast cancer. Eur J Cancer. 2003; 39:257-64.

17. Maeda K, Hamada J, Takahashi Y, Tada M, Yamamoto Y, Sugihara T, Moriuchi T. Altered expressions of HOX genes in human cutaneous malignant melanoma. Int J Cancer. $2005 ; 114: 436-41$. 
18. Zhao Y, Yamashita T, Ishikawa M. Regulation of tumor invasion by HOXB13 gene overexpressed in human endometrial cancer. Oncol Rep. 2005; 13:721-6.

19. Jung C, Kim RS, Zhang H, Lee SJ, Sheng H, Loehrer PJ, Gardner TA, Jeng MH, Kao C. HOXB13 is downregulated in colorectal cancer to confer TCF4-mediated transactivation. Br J Cancer. 2005; 92:2233-9.

20. Ewing CM, Ray AM, Lange EM, Zuhlke KA, Robbins CM, Tembe WD, Wiley KE, Isaacs SD, Johng D, Wang Y, Bizon C, Yan G, Gielzak M, Partin AW, Shanmugam V, Izatt $\mathrm{T}$, Sinari S, Craig DW, Zheng SL, Walsh PC, Montie JE, Xu J, Carpten JD, Isaacs WB, Cooney KA. Germline mutations in HOXB13 and prostate-cancer risk. N Engl J Med. 2012; 366:141-9.

21. Breyer JP, Avritt TG, McReynolds KM, Dupont WD, Smith JR. Confirmation of the HOXB13 G84E germline mutation in familial prostate cancer. Cancer Epidemiol Biomarkers Prev. 2012; 21:1348-53.

22. Akbari MR, Trachtenberg J, Lee J, Tam S, Bristow R, Loblaw A, Narod SA, Nam RK. Association between germline HOXB13 G84E mutation and risk of prostate cancer. J Natl Cancer Inst. 2012; 104:1260-2.

23. Karlsson R, Aly M, Clements M, Zheng L, Adolfsson J, Xu J, Grönberg H, Wiklund F. A population-based assessment of germline HOXB13 G84E mutation and prostate cancer risk. Eur Urol. 2014; 65:169-76.

24. Gudmundsson J, Sulem P, Gudbjartsson DF, Masson G, Agnarsson BA, Benediktsdottir KR, Sigurdsson A, Magnusson OT, Gudjonsson SA, Magnusdottir DN, et al. A study based on whole-genome sequencing yields a rare variant at 8q24 associated with prostate cancer. Nat Genet. 2012; 44:1326-9.

25. Akbari MR, Kluźniak W, Rodin R, Li S, Wokołorczyk D, Royer R, Kashyap A, Menkiszak J, Lubinski J, Narod SA, Cybulski C. The HOXB13 p.Gly84Glu mutation is not associated with the risk of breast cancer. Breast Cancer Res Treat. 2012; 136:907-9.

26. Chen Z, Greenwood C, Isaacs WB, Foulkes WD, Sun J, Zheng SL, Condreay LD, Xu J. The G84E mutation of HOXB13 is associated with increased risk for prostate cancer: results from the REDUCE trial. Carcinogenesis. 2013; 34:1260-4.

27. Xu J, Lange EM, Lu L, Zheng SL, Wang Z, Thibodeau SN, Cannon-Albright LA, Teerlink CC, Camp NJ, Johnson AM, et al. International Consortium for Prostate Cancer Genetics. HOXB13 is a susceptibility gene for prostate cancer: results from the International Consortium for Prostate Cancer Genetics (ICPCG). Hum Genet. 2013; 132:5-14.

28. Kluźniak W, Wokołorczyk D, Kashyap A, Jakubowska A, Gronwald J, Huzarski T, Byrski T, Dębniak T, Gołąb A, Gliniewicz B, et al. Polish Hereditary Prostate Cancer
Consortium. The G84E mutation in the HOXB13 gene is associated with an increased risk of prostate cancer in Poland. Prostate. 2013; 73:542-8.

29. Laitinen VH, Wahlfors T, Saaristo L, Rantapero T, Pelttari LM, Kilpivaara O, Laasanen SL, Kallioniemi A, Nevanlinna H, Aaltonen L, et al. HOXB13 G84E mutation in Finland: population-based analysis of prostate, breast, and colorectal cancer risk. Cancer Epidemiol Biomarkers Prev. 2013; 22:452-60.

30. Stott-Miller M, Karyadi DM, Smith T, Kwon EM, Kolb S, Stanford JL, Ostrander EA. HOXB13 mutations in a population-based, case-control study of prostate cancer. Prostate. 2013; 73:634-41.

31. Witte JS, Mefford J, Plummer SJ, Liu J, Cheng I, Klein EA, Rybicki BA, Casey G. HOXB13 mutation and prostate cancer: studies of siblings and aggressive disease. Cancer Epidemiol Biomarkers Prev. 2013; 22:675-80.

32. Akbari MR, Anderson LN, Buchanan DD, Clendenning M, Jenkins MA, Win AK, Hopper JL, Giles GG, Nam R, Narod S, Gallinger S, Cleary SP. Germline HOXB13 p.Gly84Glu mutation and risk of colorectal cancer. Cancer Epidemiol. 2013; 37:424-7.

33. Albitar F, Diep K, Ma W, Albitar M. Synonymous Polymorphisms in HOXB13 as a Protective Factor for Prostate Cancer. J Cancer. 2015; 6:409-11.

34. Kote-Jarai Z, Mikropoulos C, Leongamornlert DA, Dadaev T, Tymrakiewicz M, Saunders EJ, Jones M, Jugurnauth-Little S, Govindasami K, Guy M, et al. UK Genetic Prostate Cancer Study Collaborators, and ProtecT Study Group. Prevalence of the HOXB13 G84E germline mutation in British men and correlation with prostate cancer risk, tumour characteristics and clinical outcomes. Ann Oncol. 2015; 26:756-61.

35. Liberati A, Altman DG, Tetzlaff J, Mulrow C, Gøtzsche PC, Ioannidis JP, Clarke M, Devereaux PJ, Kleijnen J, Moher D. The PRISMA statement for reporting systematic reviews and meta-analyses of studies that evaluate healthcare interventions: explanation and elaboration. BMJ. 2009; 339:b2700.

36. Cochran WG. The combination of estimates from different experiments. Bio-metrics. 1954; 10:101-29.

37. Higgins JP, Thompson SG, Deeks JJ, Altman DG. Measuring inconsistency in meta-analyses. BMJ. 2003; 327:557-60.

38. Mantel N, Haenszel W. Statistical aspects of the analysis of data from retrospective studies of disease. J Natl Cancer Inst. 1959; 22:719-48.

39. DerSimonian R, Kacker R. Random-effects model for metaanalysis of clinical trials: an update. Contemp. Contemp Clin Trials. 2007; 28:105-14. 